\title{
QCD with dynamical Wilson fermions at $\beta=5.5^{*}$
}

\author{
K.M. Bitar, R.G. Edwards, U.M. Heller, and A.D. Kennedy ${ }^{\mathrm{a}}$ \\ ${ }^{a}$ SCRI, The Florida State University, Tallahassee, FL 32306-4052, USA
}

We study QCD with two flavors of dynamical Wilson fermions at $\beta=5.5$ and three values of $\kappa$. The corresponding pion masses are $0.375,0.324$ and 0.262 in lattice units, with pion to rho mass ratios of $0.76,0.71$ and 0.62 , respectively. We use the configurations to compute the heavy quark potential, leading to lattice spacings of $0.110,0.105$ and $0.099 \mathrm{fm}$, and to compute spectroscopy for several different valence quark $\kappa$ 's.

\section{INTRODUCTION}

Of the two popular unimproved ways of discretizing the Dirac operator simulations with dynamical Wilson fermions still lag behind simulations with dynamical staggered fermions. The best completed simulation with Wilson fermions, by the HEMCGC collaboration [1], was done at stronger couplings, i.e., larger lattice spacing, than the state-of-the-art staggered simulations 2:2:3], and at larger $m_{\pi} / m_{\rho}$ ratios, 0.72 and 0.60 . The lowest $m_{\pi} / m_{\rho}$ ratio achieved to date with staggered fermions, in contrast, is 0.33 [4], albeit at quite a strong coupling. There are many indications that the Wilson fermion HEMCGC simulations were done far from the continuum limit: for example $a^{-1}$, in the chiral limit, was estimated as $1600 \mathrm{MeV}$ from the nucleon mass, $1800 \mathrm{MeV}$ from the rho mass and $2000 \mathrm{MeV}$ from $f_{\pi}$, and a computation of $\Delta \beta$ proved impossible [5].

About 3 years ago, the SCRI lattice group decided to push dynamical Wilson fermion simulations to a somewhat weaker coupling with runs on the SCRI CM-2. Being limited, for technical reasons, to lattice sizes that are a power of two, we chose a $16^{3} \times 32$ lattice and a gauge coupling $\beta=5.5$. With these choices we hoped that the finite size effects will be relatively small. We have made runs at three different $\kappa$ values, to allow chiral fits and thus to test the chiral extrapolations. In each case we have about 2000 trajectories, after thermalization, with parameters given

${ }^{*}$ Preprint FSU-SCRI-96-59. To appear in the proceedings of Lattice '96, St. Louis, Missouri, USA, 4-8 June. Presented by U. M. Heller.
Table 1

Summary of run parameters

\begin{tabular}{|c|cccc|}
\hline$\kappa$ & $d t$ & $R$ & Acc & $\left\langle N_{C G}\right\rangle$ \\
\hline 0.1596 & 0.01754 & $3 \cdot 10^{-7}$ & $75 \%$ & 270 \\
0.1600 & 0.01515 & $3 \cdot 10^{-7}$ & $75 \%$ & 380 \\
0.1604 & 0.007 & $3 \cdot 10^{-7}$ & $90 \%$ & 450 \\
\hline
\end{tabular}

Table 2

Some results

\begin{tabular}{|c|cccc|}
\hline$\kappa$ & $\left\langle\frac{1}{3} \operatorname{Tr} U_{p}\right\rangle$ & $\alpha_{V}\left(\frac{3.41}{a}\right)$ & $\tau_{\text {plaq }}$ & $\tau_{\pi(10)}$ \\
\hline 0.1596 & $0.55928(9)$ & 0.178 & 33 & 65 \\
0.1600 & $0.56025(5)$ & 0.177 & 9 & 15 \\
0.1604 & $0.56131(8)$ & 0.176 & 21 & 21 \\
\hline
\end{tabular}

in Table 1. Table 2 contains some results, including rough estimates of integrated autocorrelation times. Sample time histories, for the run with $\kappa=0.1600$, are shown in Fig. 1 .

\section{THE HEAVY QUARK POTENTIAL}

On the ensembles of configurations generated, we computed the heavy quark potential from a combination of on- and off-axis timelike Wilson loops, with "APE smeared" links in the space directions [6] to enhance the signal to noise ratio. Since string breaking is estimated to occur at a distance somewhat larger than accessible to us (and we do not observe any sign of it) we used the same ansatz that is common in quenched simulations for fitting the potential

$V(\vec{r})=V_{0}+\sigma r-\frac{e}{r}-e^{\prime}\left(G_{L}(\vec{r})-\frac{1}{r}\right) ;$ 


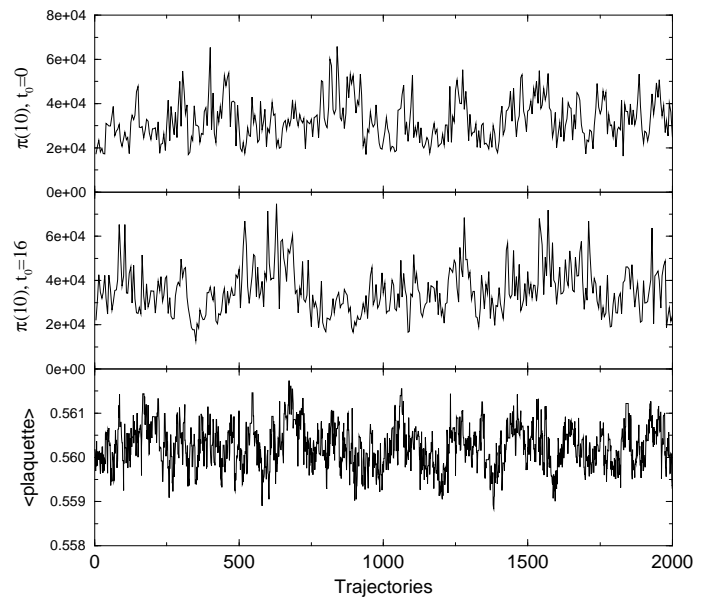

Figure 1. Time history of the plaquette and the pion correlation function at distance 10 with wall source at $t_{0}=0$ and 10 for $\kappa=0.1600$.

with $G_{L}$, the lattice Coulomb potential, taking account of the lattice artefacts present at small distances. We used fully correlated fits with the covariance matrix estimated by a jackknife method.

The parameters for the best fits, defined as in ref. [7], are listed in Table 3. We show the potentials together with the fits, in physical units as determined from the string tension, in Fig. 2. Also included in the plot are the potentials from the $\beta=5.3$ simulations as computed in ref. [7]. All curves agree fairly well, showing approximate scaling of the potential. The Coulomb coefficient $e$ is somewhat larger than for quenched calculations at comparable lattice spacing, which can be attributed to the effect of the dynamical fermions [8.

\section{SPECTROSCOPY}

For each ensemble we computed the hadron spectroscopy with six different valence quark kappas, $\kappa_{V}=\kappa_{\text {sea }}, 0.1590,0.1580,0.1395,0.1230$ and 0.1050 . The last three are quite heavy, in the $c$ to $b$ quark range, and will not be discussed here. Mesons were computed for all pairs of $\kappa_{V}$ 's, while baryons only for equal valence quarks. Mass fits, preferably to two states, and chiral fits were fully

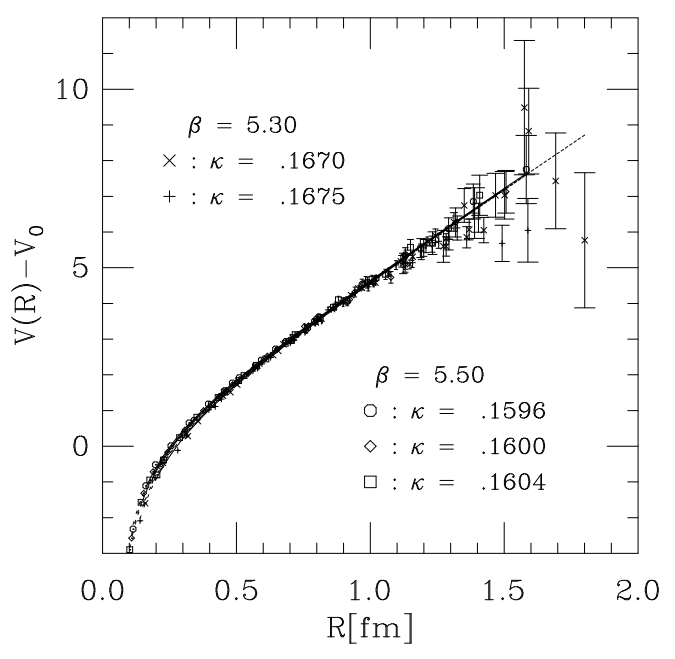

Figure 2. The potential, in physical units, including, for comparison, the $\beta=5.3$ data from [7].

correlated. In Fig. 3 we show chiral plots (vs. $1 / \kappa)$ for the results with $\kappa_{\text {sea }}=0.1600$. Similar plots for the other sea quarks and tables of all the masses can be found in ref. [9].

Having ensembles with three different sea quark masses, we can also make chiral fits for spectroscopy involving only $\kappa_{V}=\kappa_{\text {sea }}$. These are shown in Fig. 4. We obtain $\kappa_{c}=0.16116(15)$ and extrapolated masses $a m_{\rho}=0.352(12), a m_{N}=$ $0.520(18)$ and $a m_{\Delta}=0.603(15)$. These numbers, as well as the results in Table 1 for $\kappa_{\text {sea }}=0.1604$, are still preliminary. We are in the process of computing propagators from another time slice.

In Table 1 we give a collection of estimates of $a^{-1}$ in $\mathrm{MeV}$, obtained from the potential, rho, nucleon and Delta masses. For the latter, at fixed $\kappa_{\text {sea }}$, the masses were extrapolated to the physical $m_{\pi} / m_{\rho}$ ratio. The last line shows the extrapolation to zero sea quark mass from the masses obtained with $\kappa_{V}=\kappa_{\text {sea }}$. As can be seen, the lattice spacing estimates still differ considerably across each line in the table and we have to conclude that there are still rather large $\mathrm{O}(a)$ effects present. It will be difficult to decrease these lattice effects by simply going to smaller lattice spacings. 
Table 3

Summary of results from fits to the effective potentials using eq. (11). The last two columns give the scale $r_{0} / a$ determined from $r_{0}^{2} F\left(r_{0}\right)=1.65$ and the dimensionless quantity $r_{0} \sqrt{\sigma}$.

\begin{tabular}{|l|l|cc|cccc|c|cc|}
\hline$\kappa$ & $T$ & $r_{\min }$ & $r_{\max }$ & $a V_{0}$ & $a^{2} \sigma$ & $e$ & $e^{\prime}$ & $Q$ & $r_{0} / a$ & $r_{0} \sqrt{\sigma}$ \\
\hline 0.1596 & 4 & 1.41 & 13.86 & $0.731(3)$ & $0.0645(7)$ & $0.315(3)$ & $0.368(12)$ & 0.90 & $4.55(2)$ & $1.155(1)$ \\
\hline 0.1600 & 4 & 1.73 & 13.86 & $0.743(5)$ & $0.0583(9)$ & $0.329(6)$ & $0.375(13)$ & 0.77 & $4.76(3)$ & $1.149(3)$ \\
\hline 0.1604 & 4 & 2.24 & 13.86 & $0.757(6)$ & $0.0510(9)$ & $0.342(5)$ & $0.53(8)$ & 0.81 & $5.06(3)$ & $1.144(4)$ \\
\hline
\end{tabular}
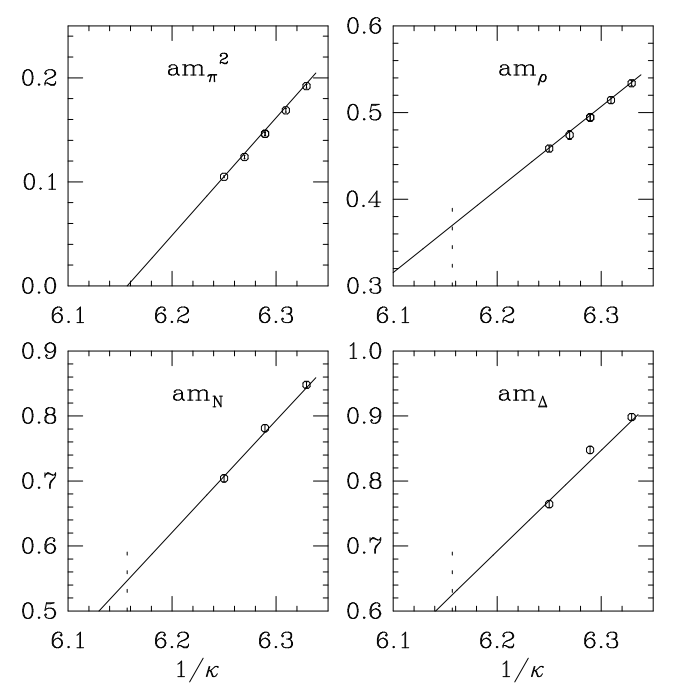

Figure 3. Chiral plots for the hadron spectroscopy on the ensemble with $\kappa_{\text {sea }}=0.1600$. The dashed lines indicate $\kappa_{c}$.
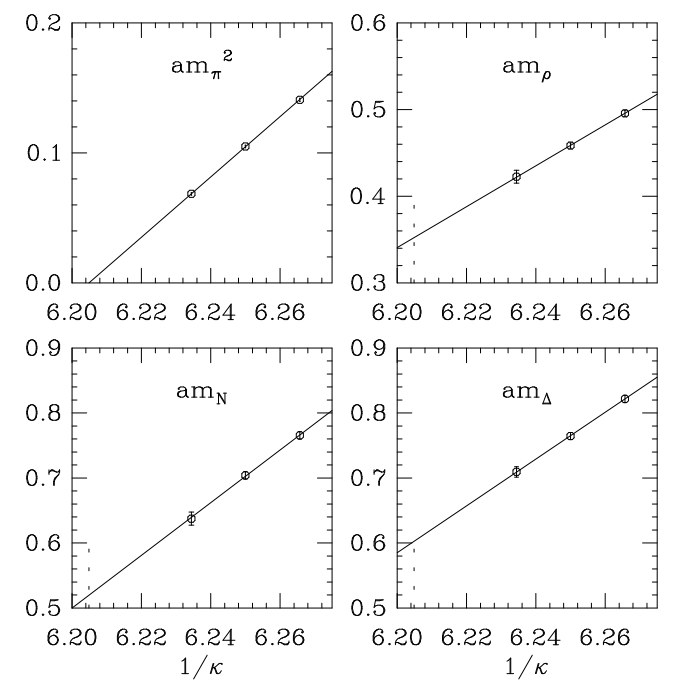

Figure 4. Chiral plots for the hadron spectroscopy with $\kappa_{V}=\kappa_{\text {sea }}$.
Table 4

Values for $a^{-1}$ in $\mathrm{MeV}$. The last line comes from extrapolations in the sea quark mass to the chiral limit.

\begin{tabular}{|c|cccc|}
\hline$\kappa$ & $r_{0}$ & $\rho$ & $N$ & $\Delta$ \\
\hline 0.1596 & $1790(10)$ & $1955(40)$ & $1650(30)$ & $1910(45)$ \\
0.1600 & $1885(15)$ & $2025(25)$ & $1685(30)$ & $1950(45)$ \\
0.1604 & $1995(15)$ & $1995(40)$ & $1755(45)$ & $1985(40)$ \\
\hline 0.16116 & $2185(25)$ & $2190(75)$ & $1805(65)$ & $2045(55)$ \\
\hline
\end{tabular}

\section{Acknowledgements}

This work was partly supported by the DOE under grants \# DE-FG05-85ER250000 and \# DE-FG05-92ER40742. The computations were carried out on the CM-2 at SCRI.

\section{REFERENCES}

1. K.M. Bitar, et al. (HEMCGC collaboration), Phys. Rev. D49 (1994) 3564.

2. F.R. Brown, et al., Phys. Rev. Lett. 67 (1991) 1062; D. Chen, Nucl. Phys. B (Proc Suppl) 42 (1995) 312 .

3. K.M. Bitar, et al. (HEMCGC collaboration), Phys. Rev. D49 (1994) 6026.

4. D. Chen and R.D. Mawhinney, Nucl. Phys. B (Proc Suppl) 34 (1994) 370.

5. K.M. Bitar, R.G. Edwards, U.M. Heller and A.D. Kennedy, FSU-SCRI-96-07, to appear in Phys. Rev. D.

6. M. Albanese, et al. (APE collaboration), Phys. Lett. 192B (1987) 163.

7. U.M. Heller, K.M. Bitar, R.G. Edwards and A.D. Kennedy, Phys. Lett. B335 (1994) 71.

8. U. Glässer, et al. (SESAM collaboration), 
hep-lat/9604014.

9. K.M. Bitar, R.G. Edwards, U.M. Heller and A.D. Kennedy, in preparation. 